\title{
ENSAYO
}

\section{Ecología de Aedes aegypti y Aedes albopictus en América y transmisión de enfermedades}

\author{
Jorge R. Rey, Philip Lounibos \\ Florida Medical Entomology Laboratory, University of Florida, Vero Beach, FL, USA
}

\begin{abstract}
La reciente expansión geográfica del mosquito Aedes albopictus ha estado acompañada en algunos sitios por la disminución en la abundancia o por la eliminación local de Aedes aegypti, pero las dos especies aún coexisten en extensas regiones de América. En este trabajo se ofrece un resumen de los posibles mecanismos responsables de sus patrones de abundancia y desplazamiento, así como de su significado en cuanto a la transmisión de enfermedades.

Entre los mecanismos observados, se encuentran la competencia por recursos, las diferencias en la capacidad para soportar la inanición, la competencia aparente a través de efectos diferenciales del parásito Ascogregarina taiwanensis, y la inhibición causada por larvas de Ae. albopictus en el desarrollo de los huevos de Ae. aegypti.

Un mecanismo propuesto como promotor de la coexistencia de estas especies es la segregación en diferentes hábitats, lo que evita la competencia directa. Aedes aegypti predomina en áreas urbanas, Ae. albopictus en áreas rurales, y las dos especies coexisten en áreas periurbanas. Existen pruebas de que la distribución de Ae. aegypti y Ae. albopictus también puede verse afectada en ciertas áreas, por la distancia desde la costa.

Otra posible causa de la rápida desaparición de Ae. aegypti es la interferencia reproductiva entre las dos especies. De acuerdo con esta hipótesis, los efectos asimétricos de los apareamientos entre especies favorecen a Ae. albopictus. Este tipo de interferencia reproductiva podría ser la causante de la eliminación de poblaciones simpátricas de las especies involucradas y de la rapidez con que $A e$. aegypti ha desaparecido de muchos lugares en América luego de la invasión de Ae. albopictus.
\end{abstract}

Palabras clave: Aedes, arbovirus, virus chikungunya, dengue, hábitat.

doi: http://dx.doi.org/10.7705/biomedica.v35i2.2514

\section{Ecology of Aedes aegypti and Aedes albopictus in the Americas and disease transmission}

The recent range expansion of the mosquito Aedes albopictus has been associated in some areas by declines in abundance or local elimination of Aedes aegypti, but the two species still coexist in large regions of the Americas. We offer a summary of the possible mechanisms responsible for the abundance and displacement pattern observed and of their significance in terms of disease transmission.

Among these mechanisms we may mention the competition for limiting resources, the differences in the ability to withstand starvation, the apparent competition through differential effects of the parasite Ascogregarina taiwanensis, and the inhibition of Ae. aegypti egg development by Ae. albopictus larvae. Habitat segregation has been proposed as a mechanism promoting the coexistence of the two species through avoidance of direct competition. Aedes aegypti predominates in urban areas, Ae. albopictus in rural ones, and both species coexist in the suburbs. There is also evidence that in certain areas, habitat segregation in terms of distance from the coast can influence the distribution of both species.

Another possible cause of the rapid disappearance of Ae. aegypti is reproductive interference between the species. According to this hypothesis, asymmetric effects of interspecific mating favor Ae. albopictus. This type of reproductive interference can result in the elimination of sympatric populations of the affected species and can be one of the major causes for the swiftness with which Ae. aegypti disappeared from some places in the Americas following invasions by Ae. albopictus.

Key words: Aedes, arboviruses, chikungunya virus, dengue, habitat.

doi: http://dx.doi.org/10.7705/biomedica.v35i2.2514

\section{Contribución de los autores:}

Ambos autores participaron en la recolección y análisis de los datos y en la escritura del artículo. 
A nivel mundial, Aedes aegypti y Aedes albopictus son dos de las especies más importantes de mosquitos, en lo que se refiere a la transmisión de enfermedades. Ambas se consideran especies invasoras, ya que han colonizado exitosamente muchos sitios fuera de sus ámbitos nativos (1). Aedes aegypti, originaria de África, migró al Nuevo Mundo durante los siglos XV al XVII a bordo de los barcos que transportaban esclavos $(2,3)$. Las múltiples invasiones de Ae. albopictus en América son de origen mucho más reciente y han ocurrido durante las últimas dos o tres décadas (4). Esta especie, originaria del Oriente e India (4), se extendió hacia el oeste hasta las islas Mauricio, las Seychelles y Madagascar, transportada por humanos hace más de un siglo, e invadió islas del Pacífico Sur cien años antes de llegar al Nuevo Mundo a bordo de buques que transportaban neumáticos (5).

Estas especies comparten varias características que les confieren ventajas adaptativas sobre otras, convirtiéndolas en invasoras exitosas (1). Una de las más importantes es que sus huevos son más o menos resistentes a la desecación, lo cual les permite sobrevivir en ambientes inhóspitos, además de favorecer su transporte por medio de humanos. Quizá de mayor importancia es su asociación con el hombre, porque las alteraciones causadas por los humanos les abren nuevos hábitats, lo que las beneficia, pues poseen características propias de especies invasoras (1). Ciertamente, esta asociación con el hombre facilita que sean transportadas, voluntaria o involuntariamente, de un lugar a otro, debido a su tendencia a alojarse en recipientes pequeños en las etapas inmaduras.

Otro rasgo importante, pero no necesariamente ventajoso, característico de todos los mosquitos, es la gran diferencia entre las etapas inmaduras (acuáticas) y los adultos (terrestres). Esto permite que diversos factores físicos (por ejemplo, la temperatura, la humedad relativa, la estructura del hábitat) y biológicos (por ejemplo, la competencia y la depredación), que ejercen efectos importantes sobre las etapas inmaduras, también influyan en la capacidad de transmitir agentes patógenos en la etapa adulta.

\footnotetext{
Correspondencia:

Jorge Rey, Florida Medical Entomology Laboratory, University of Florida, 200 9th Street SE, Vero Beach, FL 32962, USA Teléfono: (772) 778 7200, extensión 136; fax: (778) 772-7205 jrey@ufl.edu

Recibido: 19/09/14; aceptado: 03/02/15
}

La reciente expansión geográfica de Ae. albopictus ha venido acompañada en algunos sitios por la disminución en la abundancia, cuando no por la eliminación local, de Ae. aegypti (6). Este fenómeno se ha asociado con la competencia entre las dos especies. Sin embargo, hoy en día, las dos todavía coexisten en extensas regiones de América.

El desplazamiento competitivo es el resultado de principios ecológicos que postulan que dos especies no pueden ocupar el mismo nicho, lo cual conlleva una reducción de la población de una de las especies debido a la competencia con la otra. A continuación ofrecemos algunas observaciones sobre los mecanismos y los efectos de la competencia entre estas dos especies invasoras y su posible influencia sobre la transmisión de enfermedades.

La competencia entre estas especies pertenecientes al mismo subgénero se facilita porque en sus etapas inmaduras ambas se desarrollan en recipientes que acumulan agua y porque se aparean durante el día cerca de fuentes de alimentación sanguínea, por ejemplo, los humanos. En el sur de Estados Unidos, la introducción de Ae. albopictus causó la rápida eliminación de Ae. aegypti en muchos sitios y la reducción de su distribución en el sureste del país, de Texas a Florida. En Florida, O'Meara, et al., estudiaron el aumento y la disminución de la abundancia de ambas especies en recipientes artificiales en diversos condados (6). Aedes albopictus se encontró por primera vez en 1986, y en 1994 se había extendido por todo el estado, con excepción de los Cayos, en el extremo sur de la Florida.

En menos de ocho años, la distribución de $A e$. aegypti se redujo a la parte sur de la península de la Florida. En muchos sitios se observó la rápida desaparición de Ae. aegypti luego de la llegada de Ae. albopictus, o una intensa disminución de sus poblaciones, especialmente en áreas rurales. Sin embargo, en otros sitios, por ejemplo en ciudades sureñas de Estados Unidos (6,7) y Brasil (8), Ae. aegypti persistió a pesar de la invasión de Ae. albopictus. Los mecanismos competitivos causantes de este rápido desplazamiento han sido motivo de muchas conjeturas, y han dado lugar a varios estudios de campo y de laboratorio.

Por el contrario, en varias regiones Ae. aegypti ha empezado a recuperarse después de muchos años de ausencia. Por ejemplo, en varios cementerios de Florida, donde se consideraba que Ae. aegypti estaba extinta, se ha empezado a recolectar nuevamente (figura 1) y en algunos sitios 

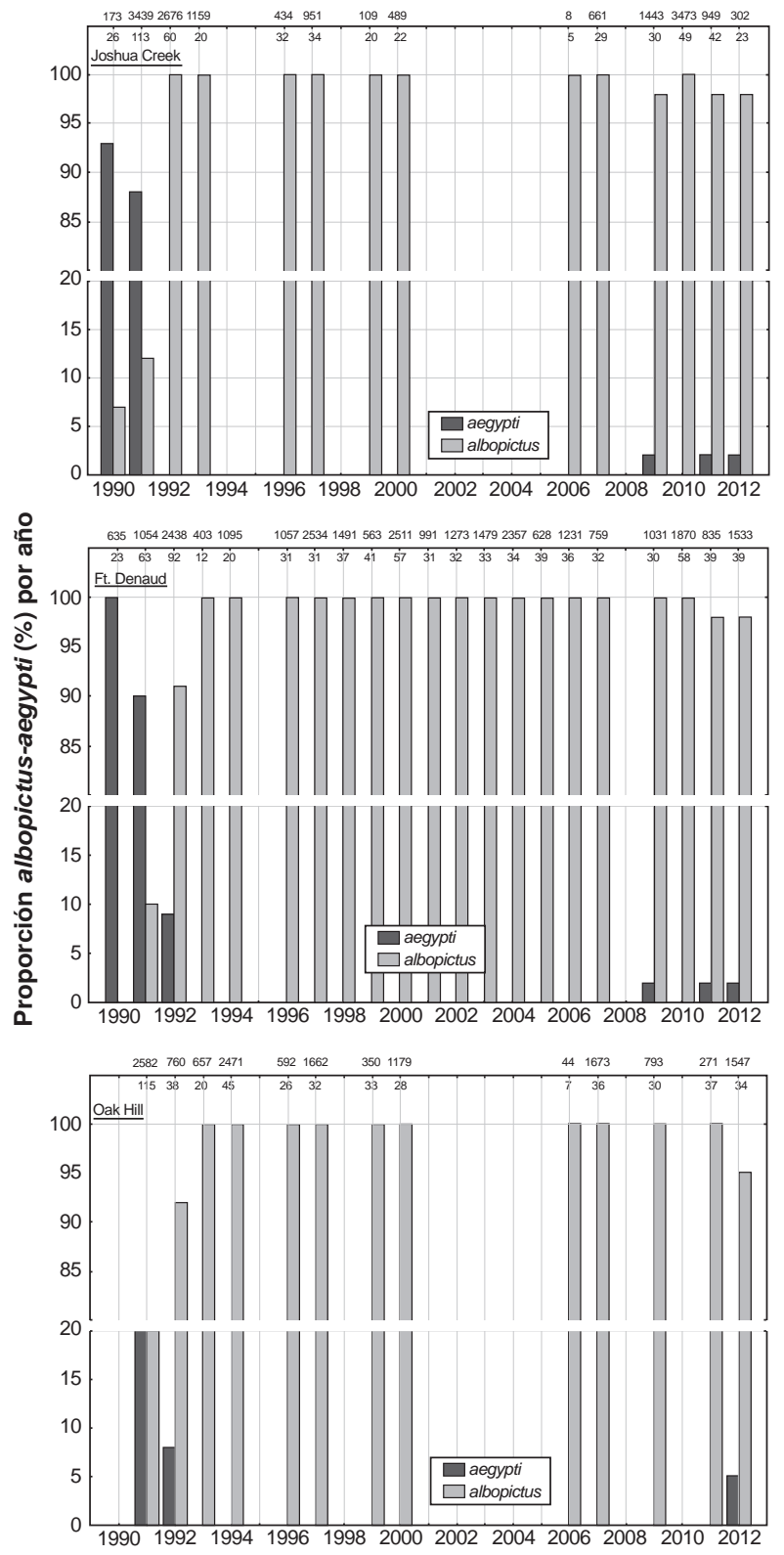

Figura 1. Abundancia relativa de Aedes aegypti y Aedes albopictus en tres cementerios rurales de Florida, Estados Unidos. Los números sobre el eje horizontal superior indican el número de muestras y los números bajo el eje corresponden al número total de larvas. Aedes albopictus reemplazó a $A$. aegypti en estos cementerios entre 1990 y 1992.

ha sobrepasado a Ae. albopictus en abundancia. Se han observado recuperaciones similares en varios sitios de la región, incluidos Colombia (9) y Argentina (10).

Es difícil establecer las distribuciones actuales de Ae. aegypti y Ae. albopictus, ya que la situación es siempre fluctuante y abundan los reportes inéditos de cambios en la distribución de ambas especies, incluidos reportes recientes de la expansión de Ae. aegypti en Argentina (10) y de Ae. albopictus en Estados Unidos (11).

\section{Mecanismos de desplazamiento y coexistencia}

\section{Segregación por hábitat}

Un mecanismo propuesto como promotor de la coexistencia entre las especies es la segregación en diferentes hábitats, lo que evita la competencia directa. Braks, et al. (8), demostraron que el hábitat ejerce una influencia importante sobre la abundancia de las dos especies; Ae. aegypti predomina en áreas urbanas, Ae. albopictus en áreas rurales, y las dos especies coexisten en áreas periurbanas de varias regiones en Brasil y en el estado de Florida. De modo similar, en la parte subtropical de dicho estado, la abundancia de las dos especies se ha relacionado con las características de los sitios muestreados en escalas de $100 \mathrm{~m}$, aproximadamente (12). Los sitios donde Ae. aegypti era más abundante, se asociaron positivamente con variables vinculadas con la urbanización y, negativamente, con variables relacionadas con ámbitos rurales. Lo opuesto se observó en sitios donde predominaba Ae. albopictus.

La segregación por hábitat a veces parece estar fomentada por elementos ajenos a la competencia. En parte, Ae. aegypti predomina en áreas urbanas porque no necesita alimentarse de néctar (azúcar), y prefiere poner los huevos, reposar y picar a humanos bajo techo (13). Por el contrario, Ae. albopictus predomina en áreas rurales porque las hembras necesitan alimentarse de néctar y prefieren poner los huevos, reposar y picar a la intemperie (4). Aunque ambas especies se asocian con los humanos, Ae. albopictus es más oportunista en la selección de fuentes de alimentación sanguínea que Ae. aegypti, que tiende a ser más dependiente de la sangre humana (14-16), aunque el fenómeno varía ampliamente de un sitio a otro. Por otro lado, las etapas inmaduras de Ae. albopictus parecen estar mejor adaptadas a los recipientes naturales que a los artificiales $(14,16)$.

Se ha observado que la distribución de Ae. aegypti y Ae. albopictus también se puede segregar en ciertas áreas de acuerdo con la distancia desde la costa. Reiskind y Lounibos (17) señalan que en Florida existe segregación de las dos especies entre hábitats costeros (donde Ae. aegypti predomina) y del interior (donde Ae. albopictus es más abundante), y establecen posibles correlaciones entre estos hábitats y factores abióticos como la 
humedad y la temperatura, así como con variables relacionadas con el hábitat, como la abundancia de recipientes con agua. El estudio no considera correlaciones con algunos factores biológicos, como la abundancia de vegetación, que también pueden influir en la distribución de estas especies.

\section{Interacción entre etapas inmaduras}

Entre los mecanismos postulados como causantes de los patrones observados de abundancia y desplazamiento, se encuentran la competencia por recursos limitados (18-20), las diferencias en la capacidad de soportar la inanición $(15,21)$, la competencia aparente mediante efectos diferentes del parásito Ascogregarina taiwanensis, que supuestamente afecta a Ae. aegypti pero no a $A$ e. albopictus (21), y la inhibición causada por larvas de Ae. albopictus en el desarrollo de los huevos de Ae. aegypti (21).

La ventaja que presenta $A$ e. albopictus para la competencia por recursos depende, sin embargo, del tipo de alimento usado en los experimentos (detrito natural, hígado molido, levadura, etc.), observándose resultados opuestos cuando se usan diferentes recursos $(1,15,19)$. En algunos estudios de campo llevados a cabo en Florida, no se pudo confirmar la competencia aparente con el parásito $A$. taiwanenesis (18). La competencia por alimentos puede explicar la eliminación de Ae. aegypti después de la invasión de Ae. albopictus en ciertas áreas, pero no explica la persistencia de Ae. aegypti en otros sitios después de la invasión de Ae. albopictus (8).

Una posible explicación es que los climas cálidos y secos favorecen a Ae. aegypti, debido a que los huevos de $A e$. albopictus son más vulnerables a condiciones de sequía que los de Ae. aegypti $(22,23)$, lo cual permite a esta última especie coexistir con Ae. albopictus. Existen datos que confirman esa observación; por ejemplo, durante la expansión de Ae. albopictus en Brasil, esta especie predominó en las áreas más húmedas y, Ae. Aegypti, en las más secas (14), en tanto que en Florida la mortalidad de los huevos de Ae. albopictus, asociada con factores microclimáticos relacionados con la temperatura y la precipitación, fueron importantes para discriminar entre sitios (cementerios) en donde Ae. aegypti desapareció luego de la llegada de Ae. albopictus y lugares donde las dos especies coexistían (23). En Calcuta, donde una importante urbanización favorece a Ae. aegypti, se observó el desplazamiento de Ae. albopictus tras la invasión de Ae. aegypti (24). En general, las dos especies coexisten en sitios más secos y cálidos, debido a la mayor mortalidad de huevos de Ae. albopictus. Estos ejemplos señalan la importancia de las interacciones entre los factores competitivos y no competitivos, a la hora de determinar la distribución local de las dos especies.

\section{Interferencia reproductiva}

Una de las primeras hipótesis planteadas para explicar la rápida desaparición de Ae. aegypti,, fue la de la interferencia reproductiva entre las dos especies. De acuerdo con esta hipótesis, los efectos asimétricos de los apareamientos entre especies favorecen a $\mathrm{Ae}$. albopictus. Pero aunque los primeros estudios demostraron dicho favorecimiento (25), otros presentaron datos contradictorios. Leahy y Craig (26) identificaron múltiples barreras contra la hibridación, pero otros autores (25) reportaron que los machos de $A e$. albopictus inseminaban a hembras de Ae. aegypti sin reparos, y que el cruce opuesto casi nunca ocurría. Los estudios de Harper y Paulson (27), sin embargo, indicaron que los cruces interespecíficos ocurrían con una frecuencia demasiado baja como para explicar los patrones de desplazamiento de Ae. aegypti por parte de Ae. albopictus.

En estudios recientes (28), se indica que ciertas sustancias de las glándulas accesorias de los machos de Ae. albopictus (que se transfieren durante el apareamiento) ejercen un fuerte efecto sobre las hembras de Ae. aegypti que les impiden apareamientos posteriores. Sin embargo, las sustancias de las glándulas accesorias de $A e$. aegypti no ejercen un efecto similar sobre las hembras de $A e$. albopictus. Este estudio fue el primero en documentar apareamientos entre $A e$. aegypti y Ae. albopictus en ambas direcciones, en poblaciones de campo en Florida. Otros estudios indican que las hembras de Ae. aegypti en simpatría con Ae. albopictus, ya sea en condiciones naturales o experimentales, desarrollan rápidamente mecanismos de aislamiento reproductivo que previenen la pérdida de potencial reproductivo en apareamientos con machos de Ae. albopictus $(29,30)$. Este tipo de interferencia reproductiva puede causar la eliminación de poblaciones simpátricas de las especies involucradas (31), y puede ser una de las principales causas de la rapidez con que Ae. aegypti desapareció del sureste de Estados Unidos y de las islas Bermudas luego de la invasión de Ae. albopictus. 
En estudios recientes, se señala que también existen interacciones entre Ae. albopictus y $A e$. aegypti durante la ovoposición. En experimentos llevados a cabo en jaulas en Florida, se notaron cambios en la selección de hábitat para depositar los huevos, así como en el número de huevos depositados por hembra y en su distribución entre varios posibles sitios de ovoposición, dependiendo de la presencia o ausencia de la otra especie (32). Por ejemplo, las hembras de Ae. aegypti depositaron más huevos en presencia de Ae. albopictus que en su ausencia, pero la presencia de las primeras no afectó el número de huevos depositados por las segundas. También, se observó que la frecuencia de oviposición en más de un sitio por parte de hembras de Ae. albopictus fue menor en presencia de Ae. aegyptique en su ausencia.

\section{Significado en la transmisión de enfermedades}

Además de ser una especie implicada en la transmisión de dengue y fiebre amarilla, Ae. aegypti también transmite otras enfermedades, incluida la fiebre de chikungunya y varios tipos de encefalitis. Es importante mencionar que el llamado mosquito de la fiebre amarilla no ha transmitido esta enfermedad en América en más de 50 años $(33,34)$, debido, en parte, a las campañas de vacunación y de control del mosquito en la región. Sin embargo, la fiebre amarilla selvática continúa manifestándose en primates no humanos en el hemisferio sur, transmitida principalmente por Haemagogus spp. Frecuentemente se reportan casos en humanos, especialmente en personas que trabajan en bosques y selvas de la región. Existe preocupación de que el retorno de Ae. aegypti a muchos centros urbanos, pueda causar el resurgimiento de la fiebre amarilla urbana. Tal podría ser el caso de lo observado en Paraguay en 2008 (35).

Aedes albopictus también puede infectarse naturalmente o en el laboratorio con numerosos arbovirus $(14,16,36)$, y tiene la capacidad de desarrollarse en recipientes artificiales y naturales. Por lo tanto, esta especie puede establecer un enlace entre formas selváticas y urbanas de varias enfermedades, incluidas la fiebre amarilla y la encefalitis equina venezolana (16). Aedes albopictus se ha implicado en la transmisión de dengue en varios sitios, entre ellos Hawái (37), Macao (38) y otros lugares donde Ae. aegypti no es común (29).

Desde que se estableció en el este de Estados Unidos, Ae. albopictus se ha involucrado en la transmisión del virus de LaCrosse en ciclos humanos y enzoóticos junto con el vector endémico Ae. triseriatus (39). Es posible que el aumento reciente de la incidencia de la enfermedad en estados ubicados en el sureste del país, se deba a la invasión de $A e$. albopictus y al declive de $A e$. aegypti, que no es un transmisor eficiente de este virus (16). Aedes albopictus también es un vector competente del virus chikungunya y, bajo ciertas circunstancias, puede ser mejor que Ae. aegypti (40). Las recientes epidemias en la región del Océano Indico, demuestran que la expansión de Ae. albopictus puede aumentar la incidencia de esta enfermedad (16).

Varios estudios de laboratorio demuestran que la tasa de infección con el virus del dengue (DENV) es mayor en Ae. albopictus que en Ae. aegypti, pero la diseminación del virus es mayor en la segunda que en la primera especie $(16,41)$. Gonçalves de Castro, et al. (42), demostraron que en Brasil las tasas de infección, diseminación y transmisión vertical de DENV-2 en Ae. albopictus fueron mayores que en $A e$. aegypti, y advierten que esta especie puede ser muy importante en el mantenimiento y la transmisión del virus en el país. En Colombia, Méndez, et al. (43), describieron uno de los primeros casos que involucraban poblaciones naturales de Ae. albopictus en la transmisión de dengue, tras un brote de la enfermedad en Buenaventura. Desde entonces, esta especie se ha implicado en la transmisión del dengue en otros países, incluidos Brasil (44), Costa Rica (C. Frederickson, comunicación personal) y México (45).

Es probable que las diferencias en el potencial para transmitir el dengue, se deriven de factores no relacionados con la capacidad vectorial, tales como la selección de fuentes de alimentación sanguínea y la abundancia local de las dos especies. Por ejemplo, en América, Ae. aegypti es mejor vector de dengue que Ae. albopictus debido, en parte, a que pica a humanos con mayor frecuencia que $A e$. albopictus. En general, la estrecha relación de Ae. aegypti con los humanos también la convierte en una transmisora más eficiente del dengue en el resto del mundo (16).

Los patrones de competencia y desplazamiento entre estas especies tienen consecuencias sobre la transmisión de enfermedades como el dengue y la fiebre de chikungunya. Las estrategias de control contra Ae. albopictus pueden dar lugar al resurgimiento de Ae. aegypti, mientras que el control de $A e$. aegypti ha permitido que Ae. albopictus colonice sitios más urbanos (21). Sin embargo, 
la baja tolerancia de $A e$. albopictus a condiciones de sequía parece limitar su presencia en los hábitats urbanos. Las diferentes características epidemiológicas de las dos especies afectan directamente la transmisión de enfermedades y sus diferencias ecológicas afectan las estrategias locales de control.

Las dos especies difieren en su capacidad para transmitir diferentes enfermedades. En el contexto actual, el desplazamiento de Ae. aegypti por parte de Ae. albopictus puede resultar en una disminución en la transmisión e incidencia del dengue, lo cual eventualmente dará lugar a una reducción en la inmunidad colectiva. El tema se complica si Ae. aegypti se restablece en el mismo sitio donde la inmunidad ha disminuido debido a su desaparición, y la transmisión de DENV aumenta nuevamente debido a que la población se torna más vulnerable. Sin embargo, debemos recordar que, excepto en Estados Unidos y las Bermudas, donde el DENV no es endémico, el desplazamiento y su frecuencia por competencia de Ae. aegypti por parte de Ae. albopictus, aún no están bien documentados.

Para principios de diciembre de 2014, la epidemia de chikungunya que actualmente azota al área del Caribe había resultado en más de 994.000 infecciones en América (46). Nuevamente, los patrones de abundancia relativa de las dos especies, ya sean causados por segregación ecológica por hábitat, competencia por recursos, interferencia reproductiva o combinaciones de varios mecanismos, pueden tener trascendencia sobre la transmisión de la enfermedad en América, ya que la importancia de ambas especies como vectores varía ampliamente dependiendo de las circunstancias ecológicas, geográficas, ambientales y epidemiológicas (47).

También se sospecha que la competencia en sí puede influir en la interacción entre los mosquitos y el virus. Por ejemplo, en trabajos de laboratorio, Alto, et al. (48), demostraron que la competencia intraespecífica o entre especies pueden aumentar la tasa de infección, los títulos de virus en el cuerpo y la diseminación del virus en Ae. albopictus, pero no los de Ae. aegypti. Esta situación es complicada porque los mosquitos pequeños son más propensos a la infección y la diseminación del DENV que los de mayor tamaño (41), pero la mayor propensión a la infección y la diseminación de vectores más pequeños pueden obviarse dada su menor vida útil (49). Debido a que la competencia tiende a disminuir el tamaño del mosquito adulto (50), se hace difícil diferenciar entre efectos directos de la competencia y efectos indirectos causados por la reducción en el tamaño de los mosquitos.

Además de los posibles efectos directos sobre la transmisión de los virus, los resultados anteriores ponen de relieve la influencia que las condiciones de desarrollo de las larvas tienen sobre la capacidad vectorial de los adultos en poblaciones locales de mosquitos.

\section{Conclusión}

Aedes aegypti y Ae. albopictus son dos de las especies de mosquitos más invasivas. Frecuentemente se enfrentan entre sí en sus zonas de distribución, ya sean nativas o invadidas, lo que a menudo causa el desplazamiento competitivo de una de las especies, como se ha documentado en el sur de los Estados Unidos, donde Ae. aegypti fue rápidamente reemplazado por Ae. albopictus a finales de la década de 1980 y principios de los 90. Ambas especies son vectores importantes de diversos virus, aunque desempeñan diferentes papeles en la transmisión y el mantenimiento de agentes patógenos humanos, como los virus del dengue y el del chikungunya.

Uno de los posibles mecanismos propuestos para explicar la exclusión competitiva, es la interferencia reproductiva asimétrica. Recientemente, la acción de este mecanismo se ha demostrado con hallazgos científicos sólidos. Sin embargo, las poblaciones de Ae. aegypti que logran mantenerse en simpatría con Ae. albopictus, desarrollan rápidamente resistencia por medio del desplazamiento debido a los caracteres reproductivos. La competencia por recursos limitados entre estas especies en sus etapas inmaduras, también puede jugar un papel en el desplazamiento espacial de una u otra, pero el resultado final depende de múltiples variables físicas y biológicas. Frecuentemente, las dos especies coexisten en la misma región por medio de la segregación en diferentes hábitats, ya sea debido a la competencia o a factores ambientales que favorecen a una u otra.

Los patrones de abundancia relativa de las dos especies, debidos a la segregación ecológica por hábitat, a la competencia por recursos, la interferencia reproductiva, o la combinación de varios mecanismos, pueden tener trascendencia sobre la transmisión de la enfermedad en América, ya que la importancia de ambas especies como vectores varía ampliamente, dependiendo de las circunstancias ecológicas, geográficas, ambientales y epidemiológicas. 


\section{Estudios futuros}

Es necesario acopiar más documentación sobre el desplazamiento competitivo entre estas especies, especialmente en los trópicos, donde son vectores importantes. Para ello se requiere una vigilancia minuciosa y cuantitativa antes, durante y después de invasiones de una u otra especie, ya que no es suficiente contar solamente con los datos sobre la presencia o ausencia de Ae. aegyptio de Ae. albopictus. Se requiere estudiar la incidencia de los apareamientos entre las dos especies en sus zonas de simpatría. También, sería ventajoso comprender más a fondo los compromisos involucrados en el desarrollo de resistencia a los apareamientos entre especies y la correspondiente pérdida de eficacia biológica. En las áreas donde coexisten, es necesario integrar los efectos de diferentes factores, incluida la competencia entre los adultos y las larvas de las dos especies, así como las preferencias ambientales propias de cada una para predecir distribuciones y abundancias locales. Por último, se necesita explorar más a fondo la relación entre los factores ecológicos y epidemiológicos en estas especies, para lograr una mejor comprensión de sus respectivos roles en la transmisión de arbovirus.

\section{Recomendaciones}

Aunque Ae. albopictus como posible vector del dengue y de otros arbovirus no iguala a Ae. aegypti, todo programa de salud pública que trate de controlar enfermedades por arbovirus potencialmente transmitidas por Ae. aegypti, debe tener en cuenta la presencia de Ae. albopictus. Esta especie es residente permanente de nuestra región $y$, desde que invadió a América, los indicios de su participación en la transmisión de enfermedades han aumentado. Las interacciones de Ae. albopictus con Ae. aegypti y con otros mosquitos pueden modificar la capacidad vectorial de las especies involucradas de modos no siempre predecibles.

\section{Conflicto de intereses}

No existen conflictos de intereses relacionados con este manuscrito.

\section{Financiación}

Este estudio fue financiado parcialmente por los National Institutes of Health (NIH) de los Estados Unidos, mediante los subsidios R01 Al044793 y R21 AI095780 a LPL.

\section{Referencias}

1. Juliano SA, Lounibos LP. Ecology of invasive mosquitoes: Effects on resident species and on human health. Ecol
Lett. 2005;8:558-74. http://dx.doi.org/10.1111/j.1461-0248. 2005.00755

2. Christophers R. Aedes aegypti: The yellow fever mosquito. Londres: Cambridge University Press; 1960.

3. Tabachnick WJ. Evolutionary genetics and arthropodborne disease: The yellow fever mosquito. Amer Entomol. 1991;37:14-24.

4. Hawley WA. The biology of Aedes albopictus. J Am Mosq Control Assoc. 1998;14(Suppl.1):1-39.

5. Benedict MQ, Levine RS, Hawley WA, Lounibos LP. Spread of the tiger: Global risk of invasion by the mosquito Aedes albopictus. Vector-Borne Zoon Dis. 2007;7:76-85. http://dx.doi.org/10.1089/vbz.2006.0562

6. O'Meara GF, Evans LF Jr., Gettman AD, Cuda JP. Spread of Aedes albopictus and decline of $A$. aegypti (Diptera: Culicidae) in Florida. J Med Entomol. 1995;32:554-62.

7. Hornby JA, Moore DE, Miller TW. Aedes albopictus distribution, abundance, and colonization in Lee County, Florida, and its effect on Aedes aegypti. J Am Mosq Control Assoc. 1994;10:397-402.

8. Braks MA, Honorio NA, Lourenço-de-Oliveira R, Juliano SA, Lounibos LP. Convergent habitat segregation of Aedes aegypti and Aedes albopictus (Diptera: Culicidae) in southeastern Brazil and Florida. J Med Entomol. 2003;40:785-94.

9. Carvajal JJ. Variação espacial e temporal dos vetores do dengue Aedes (Stegomyia) albopictus (Skuse, 1894) e Aedes (Stegomyia) aegypti (Linnaeus, 1762) na área urbana do município de Letícia (Amazonas-Colômbia) e sua associação com a transmissão do dengue na tríplice fronteira amazônica (Colômbia-Brasil-Peru). Dissertação de Mestrado em Medicina Tropical. Rio de Janeiro: Instituto Oswaldo Cruz, Fundação Oswaldo Cruz; 2013. p. 126.

10. Vezzani D, Carbajo A. Aedes aegypti, Aedes albopictus, and dengue in Argentina: Current knowledge and future directions. Mem Inst Oswaldo Cruz. 2008;103:66-74. http:// dx.doi.org/10.1590/S0074-02762008005000003

11. Gloria-Soria A, Brown JE, Kramer V, Hardstone Yoshimizu M, Powell JR. Origin of the dengue fever mosquito, Aedes aegypti, in California. PLoS Negl Trop Dis. 2014;8:e3029. http://dx.doi.org/10.1371/journal.pntd.0003029.

12. Rey JR, Nishimura N, Wagner B, Braks MA, O'Connell SM, Lounibos LP. Habitat segregation of mosquito arbovirus vectors in south Florida. J Med Entomol. 2006;43:1134-41.

13. Harrington LC, Edman JD, Scott TW. Why do female Aedes aegypti (Diptera: Culicidae) feed preferentially and frequently on human blood? J Med Entomol. 2001;38: 411-22.

14. Marques CC, Marques GR, DeGallier N. Is Aedes albopictus only a pest mosquito or also a vector of arboviruses in Brazil. En: Travassos da Rosa AP, Vasconcelos PF, Travassos da Rosa JF, editores. An overview of arbovirology in Brazil and neighboring countries. Belém: Instituto Evandro Chagas; 1998. p. 248-60.

15. Barrera R. Competition and resistance to starvation in larvae of container-inhabiting Aedes mosquitoes. Ecol Entomol. 1996;21:117-27. http://dx.doi.org/10.1111/j.13652311.1996.tb01178.x 
16. Lambrechts L, Scott TW, Gubler DJ. Consequences of the expanding global distribution of Aedes albopictus for dengue virus transmission. PLoS Negl Trop Dis. 2010;4:e646. http:// dx.doi.org/10.1371/journal.pntd.0000646.

17. Reiskind MH, Lounibos LP. Spatial and temporal patterns of abundance of Aedes aegypti L. (Stegomyia aegypti) and Aedes albopictus (Skuse) [Stegomyia albopictus (Skuse)] in southern Florida. Med Vet Entomol. 2013;27:421-9. http:// dx.doi.org/10.1111/mve.12000

18. Juliano SA. Species introduction and replacement among mosquitoes: Interspecific resource competition or apparent competition? Ecology. 1998;79:255-68. http://dx.doi.org/10. 1890/0012-9658(1998)079[0255:SIARAM]2.0.CO;2

19. Juliano SA. Species interactions among larval mosquitoes: Context dependence across habitat gradients. Annu Rev Entomol. 2009;54:37-56. http://dx.doi.org/10.1146/annurev. ento.54.110807.090611

20. Braks MA, Honório NA, Lounibos LP, Oliveira RL, Juliano SA. Interspecific competition between two invasive species of container mosquitoes, Aedes aegypti and Aedes albopictus (Diptera: Culicidae), in Brazil. Ann Entomol Soc Am. 2004;97:130-9.

21. Lounibos LP. Competitive displacement and reduction. En: Floore TE, Becnel J, editores. Biorational control of mosquitoes. Bull Amer Mosq Control Assoc. 2007;23:276-82.

22. Juliano SA, O'Meara GF, Morrill JR, Cutwa MM. Desiccation and thermal tolerance of eggs and the coexistence of competing mosquitoes. Oecologia. 2002;130:458-69. http:// dx.doi.org/10.1007/s004420100811

23. Lounibos L, O'Meara G, Juliano S, Nishimura N, Escher $\mathbf{R}$, Reiskind $\mathbf{M}$, et al. Differential survivorship of invasive mosquito species in South Florida cemeteries: Do site specific microclimates explain patterns of coexistence and exclusion? Ann Entomol Soc Am. 2010;103:757-70. http:// dx.doi.org/10.1603/AN09142

24. Gilotra SK, Rozeboom LE, Bhattacharya NC. Observations on possible competitive displacement between populations of Aedes aegypti Linnaeus and Aedes albopictus Skuse in Calcutta. Bull World Health Org. 1967;37:437-46.

25. Nasci, RS, Hare, SG, Willis, FS. Interspecific matings between Louisiana strains of Aedes albopictus and Aedes aegypti in the field and in laboratory. J Am Mosq Control Assoc. 1989;5:416-21.

26. Leahy MG, Craig GB Jr. Barriers to hybridization between Aedes aegypti and Aedes albopictus (Diptera: Culicidae). Evolution. 1967;21:41-58.

27. Harper J, Paulson SL. Reproductive isolation between Florida strains of Aedes aegypti and Aedes albopictus. J Amer Mosq Control Assoc. 1994;10:88-92.

28. Tripet F, Lounibos LP, Robbins D, Moran J, Nishimura N, Blosser EM. Competitive reduction by satyrization? Evidence for interspecific mating in nature and asymmetric reproductive competition between invasive mosquito vectors. Am J Trop Med Hyg. 2011;85:265-70. http://dx.doi. org/10.4269/ajtmh.2011.10-0677.

29. Bargielowski IE, Lounibos LP, Carrasquilla MC. Evolution of resistance to satyrization through reproductive character displacement in populations of invasive dengue vectors. Proc Natl Acad Sci USA. 2013;110:2888-92. http://dx.doi. org/10.1073/pnas.1219599110
30. Bargielowski I, Lounibos LP. Rapid evolution of reduced receptivity to interspecific mating in the dengue vector Aedes aegypti in response to satyrization by invasive Aedes albopictus. Evol Ecol. 2014;28:193-203. http://dx.doi. org/10.1007/s10682-013-9669-4

31. Ribeiro JM, Spielman A. The satyr effect: A model predicting parapatry and species extinction. Amer Natur. 1986;128:513-28.

32. Rey JR, O'Connell SC. Oviposition by $A$. aegypti and $A$. albopictus: Influence of congeners and of oviposition site characteristics. J Vector Ecol. 2014;39:1-7. http://dx.doi. org/10.1111/j.1948-7134.2014.12086.x.

33. Barrett AD, Higgs S. Yellow fever: A disease that has yet to be conquered. Annu Rev Entomol. 2007;52:209-29. http:// dx.doi.org/10.1146/annurev.ento.52.110405.091454

34. Gardner CL, Ryman KD. Yellow fever: A reemerging threat. Clin Lab Med. 2010;30:237-60. http://dx.doi.org/10.1016/j. cll.2010.01.001.

35. Johansson MA, Arana-Vizcarrondo N, Biggerstaff BJ, Gallagher N, Marano N, Staples JE. Assessing the risk of international spread of yellow fever virus: A mathematical analysis of an urban outbreak in Asunción, 2008. Am J Trop Med Hyg. 2012;86:349-58. http://dx.doi.org/10.4269/ ajtmh.2012.11-0432

36. Mitchell CJ, Miller B, Gubler DJ. Vector competence of Aedes albopictus from Houston, Texas, for dengue serotype-1 to serotype-4, yellow fever, and Ross River viruses. J Amer Mosquito Control Assoc. 1987;3:460-5.

37. Effler PV, Pang L, Kitsutani P, Vorndam V, Nakata M, Ayers T, et al. Dengue fever, Hawaii, 2001-2002. Emerg Infect Dis. 2005;11:742-9. http://dx.doi.org/10.3201/eid1105. 041063

38. Almeida AP, Baptista SS, Sousa CA, Novo MT, Ramos HC, Panella NA, et al. Bioecology and vectorial capacity of Aedes albopictus (Diptera: Culicidae) in Macao, China, in relation to dengue virus transmission. J Med Entomol 2005;42:419-28.

39. Gerhardt RR, Gottfried KL, Apperson CS, Davis BS, Erwin PC, Smith AB, et al. First isolation of La Crosse virus from naturally infected Aedes albopictus. Emerg Infect Dis. 2001;7:807-12. http://dx.doi.org/10.3201/eid0705.017506

40. Vazeille M, Moutailler S, Coudrier D, Rousseaux C, Khun $\mathbf{H}$, Huerre $\mathbf{M}$, et al. Two chikungunya isolates from the outbreak of La Reunion (Indian Ocean) exhibit different patterns of infection in the mosquito, Aedes albopictus. Plos One. 2007;2: e1168. http://dx.doi.org:10.371/journal. pone0001168

41. Alto BW, Reiskind MH, Lounibos LP. Size alters susceptibility of vectors to dengue virus infection and dissemination. Am J Trop Med Hyg. 2008;79:688-95.

42. Gonçalves de Castro M, Nogueira RM, Schatzmayr HG, Miagostovich M, Lourenco-de-Oliveira R. Dengue virus detection by using reverse transcription-polymerase chain reaction in saliva and progeny of experimentally infected Aedes albopictus from Brazil. Mem Inst Oswaldo Cruz. 2004;99:809-14. http://dx.doi.org/10.1590/S007402762004000800005

43. Méndez F, Barreto M, Arias JF, Rengifo G, Muñoz J, Burbano ME, et al. Human and mosquito infections by 
dengue viruses during and after epidemics in a dengueendemic region of Colombia. Am J Trop Med Hyg. 2006;74:678-83.

44. de Figueiredo ML, Gomes AD, Amarilla AA, Leandro AD, Orrico AD, de Araujo RF, et al. Mosquitoes infected with dengue viruses in Brazil. Virol J. 2010;7:152 http://dx.doi. org/10.1186/1743-422X-7-152.

45. Ibáñez-Bernal S, Briseño B, Mutebi JP, Argot E, Rodríguez G, Martínez-Campos C, et al. First record in America of Aedes albopictus naturally infected with dengue virus during the 1995 outbreak at Reynosa, Mexico. Med Vet Entomol. 1997;11:305-9.

46. Pan American Health Organization/World Health Organization. Número de casos reportados de chikungunya en países o territorios de las Américas 2013-2014. Semana Epidemiológica/SE 49. Fecha de consulta: 8 de diciembre de 2014. Disponible en: http://www.paho.org/hq/index. php?option=com_docman\&task=doc_download\&ltemid=\& gid=28447\&lang=en.
47. Weaver SC. Arrival of Chikungunya virus in the New World: Prospects for spread and impact on public health. PLoS Negl Trop Dis. 2014;8:e2921. http://dx.doi.org/10.1371/ journal.pntd.0002921

48. Alto BW, Lounibos LP, Mores CN, Reiskind MH. Larval competition alters susceptibility of adult Aedes mosquitoes to dengue infection. Proc Biol Sci. 2008;275:463-71. http:// dx.doi.org/10.1098/rspb.2007.1497

49. Juliano SA, Ribeiro GS, Maciel-de-Freitas R, Castro MG, Codeço C, Lourenço de Oliveira R, et al. She's a femme fatale: Low-density larval development produces good disease vectors. Mem Inst Oswaldo Cruz. 2014;109:96-103. http://dx.doi.org/10.1590/0074-02760140455

50. Mitchell Foster K, Ma BO, Warsame-Ali S, Logan C, Rau ME, Lowenberger $\mathbf{C}$. The influence of larval density, food stress, and parasitism on the bionomics of the dengue vector Aedes aegypti (Diptera: Culicidae): Implications for integrated vector management. J. Vector Ecol. 2012;37:221-9. http://dx.doi.org/10.1111/j.1948-7134.2012.00220.x 